\title{
POROUS SETS AND CONVERGENCE OF FOURIER SERIES
}

\author{
CASPER GOFFMAN
}

(Communicated by Christopher D. Sogge)

\begin{abstract}
In this note, examples are given of a continuous function whose graph is porous but whose Fourier series diverges at a point and of a continuous function whose graph is not porous but whose Fourier series converges uniformly.
\end{abstract}

About five years ago we asked whether or not the graph of Brownian motion is, with probability 1 , a porous set in the plane. The question stimulated substantial interest and was answered affirmatively by Cox and Griffin [CG]. A second topic concerning porosity of planar graphs of continuous functions relates to the uniform convergence of the Fourier series of the function. A preliminary report was given at the classical analysis section of the first joint meeting of the LMS and AMS in Cambridge, England on July 1, 1992.

We define porous and $\sigma$ porous sets. Let $R^{2}$ be the cartesian plane with rectangular coordinates $(x, y)$. We consider oriented squares, i.e., squares whose sides are parallel to the coordinate axes. Let $S \subset R^{2}$ and $p \in S$. We consider squares $Q_{p}$ with center at $p$. Let $\left|Q_{p}\right|$ be the area of $Q_{p}$, and let $\alpha\left(Q_{p}\right)$ be the supremum of $|Q|$, with $Q \subset Q_{p}$ and $Q \cap S=\varnothing . S$ is said to be porous at $p$ if

$$
\limsup _{\left|Q_{p}\right| \rightarrow 0} \frac{\alpha\left(Q_{p}\right)}{\left|Q_{p}\right|}>0 .
$$

$S$ is a porous set if it is porous at every $p \in S$.

$S$ is said to be $\sigma$ porous if it is the union of countably many porous sets. If $D$ is the class of countable sets, $P$ the class of $\sigma$ porous sets, $Z$ the class of sets of measure zero, and $C$ the class of sets of the first category of Baire, then $D \subset P, P \subset Z$, and $P \subset C$. In many cases where the exceptional set was known to be in $Z$ or in $C$, it is now known to be in $P$.

In this note, we give an example of a continuous function whose graph is a porous set but whose Fourier series diverges at some points, and an example of a continuous function whose graph is not porous but whose Fourier series converges uniformly.

We review classes of functions of generalized bounded variation, which are useful to us, and whose Fourier series converges uniformly.

Received by the editors February 14, 1994 and, in revised form, May 12, 1994.

1991 Mathematics Subject Classification. Primary 42A16, 26A15.

@1 1995 American Mathematical Society 
Recall that a continuous function $f:[-\pi, \pi] \rightarrow R$ is of bounded variation if $\sup \sum_{i=0}^{n-1}\left|f\left(x_{i+1}\right)-f\left(x_{i}\right)\right|<\infty$, for all partitions $-\pi \leq x_{0}<x_{1}<\cdots \leq$ $x_{n}=\pi$ of $[-\pi, \pi]$. Let $1 B V$ be the set of functions of bounded variations. A continuous function $f$ is said to be in $2 B V$ if $\sup \sum_{i=0}^{n-1}\left|f\left(x_{i+1}\right)-f\left(x_{i}\right)\right|^{2}<\infty$. This class was introduced by $\mathrm{N}$. Wiener who showed that if $f$ is continuous and in $2 B V$, then its Fourier series converges uniformly. Let $p \geq 1 . p B V$ is the set of continuous functions for which $\sup \sum_{i=0}^{n-1}\left|f\left(x_{i+1}\right)-f\left(x_{i}\right)\right|^{p}<\infty$. L. C. Young [Y] showed that if $f \in p B V$, then its Fourier series converges uniformly. A more general class of functions was introduced implicitly in [G] and was labelled HBV, of harmonic bounded variation in [W], where its properties were developed. Let $I_{n}=\left[a_{n}, b_{n}\right], n=1,2, \ldots$, be a pairwise disjoint sequence of intervals in $[-\pi, \pi] . f \in H B V$ if it is continuous and if

$$
\sup \sum_{n=1}^{\infty} \frac{1}{n}\left|f\left(b_{n}\right)-f\left(a_{n}\right)\right|<\infty .
$$

It is known that $H B V \supset p B V$ for every $p \geq 1$ and that the Fourier series converges uniformly for every $f \in H B V$.

We construct a continuous periodic function, of period $2 \pi$ on $[-\pi, \pi]$, whose graph is not porous, such that $f \in p B V$, for some $p \geq 1$, so that its Fourier series converges uniformly. We use the function $y=x^{\overline{1} / 2}, x>0$, and its reflections about the $x$-axis, the $y$-axis, and the origin, as a guide to our construction. We first define $f(x)$ for $0 \leq x \leq 1$. For every $n=1,2, \ldots$, define $f(x)$ on $I_{n}=\left[\frac{1}{n+1}, \frac{1}{n}\right]$, as follows. Let $f\left(\frac{1}{n+1}\right)=f\left(\frac{1}{n}\right)=0$. For $m=0, \ldots, n-1$, let $I_{n m}=\left[\frac{1}{n+1}+\frac{m}{n(n+1)}, \frac{1}{n+1}+\frac{m+1}{n(n+1)}\right]$. Define $f$ on $I_{n m}$ as follows. Divide $I_{n m}=[\alpha, \beta]$ into four equal parts by the points $a_{1}=\alpha$, $a_{2}=\alpha+\frac{\beta-\alpha}{4}, a_{3}=\alpha+\frac{\beta-\alpha}{2}, a_{4}=\alpha+\frac{3(\beta-\alpha)}{4}, a_{5}=\beta$. Let $f\left(a_{1}\right)=f\left(a_{3}\right)=$ $f\left(a_{5}\right)=0, f\left(a_{2}\right)=\frac{1}{(n+1)^{1 / 2}}, f\left(a_{4}\right)=-\frac{1}{(n+1)^{1 / 2}}$, and join these five consecutive points linearly. Define $f(x)$ on $[-1,0]$ by reflection about the $y$-axis. Extend $f(x)$ as 0 on $[-\pi,-1]$ and $[1, \pi]$. For $p \geq 1$, the $p$ variation of $f(x)$ is $C \sum_{n=1}^{\infty} \frac{n}{(n+1)^{p / 2}} \leq C \sum_{n=1}^{\infty} \frac{1}{(n+1)^{p / 2-1}}$ which converges if $\frac{p}{2}-1>1$ or $p>4$. In particular, $f \in 5 B V$ so that its Fourier series converges uniformly; we show that the graph of $f(x)$ is not porous. Let $a \in I_{n}$, and let $Q$ be the square whose sides are $x= \pm a, y= \pm a$. Let $S$ be the graph of $f(x)$. By studying the graph of $f(x)$ we observe that $\alpha(Q) \leq\left(\frac{2 a}{n}\right)^{2}$. It follows that $S$ is not porous at $(0,0)$.

We indicate an example of a nonnegative continuous function $f$ on $[-\pi, \pi]$, periodic of period $2 \pi$, whose graph is porous and whose Fourier series diverges at $x=0$. It is a slight modification of a standard example [R]. Let $\left\{I_{n}\right\}$ be a sequence of pairwise disjoint closed intervals, such that $I_{n+1}$ is to the right of 0 and to the left of $I_{n}$ and the left endpoints of the $I_{n}$ converge to 0 . For every $n$, let $J_{n}$ be the closed interval whose left end point is the right end point of $I_{n+1}$ and whose right end point is the left end point of $I_{n}$. Let $f$ be 0 on $[-\pi, 0]$ and on each $J_{n}$. On each $I_{n}$, let $f$ be highly oscillatory, bounded by $\frac{1}{n}$, and such that $\int_{I_{n}}\left|D_{k_{n}}(t)\right| f(t) d t>n$, for some $k_{n}$, where $\left\{k_{n}\right\}$ is an increasing sequence. The Fourier series of $f$ diverges at $x=0$. The porosity of the graph is obtained by choosing $I_{n}$ and $J_{n}$, for every $n$, so that the length of $J_{n}$ is more than $n$ times that of $I_{n}$ for every $n$. 
The following related question seems to be difficult. Is there a continuous function whose graph is not $\sigma$ porous but whose Fourier series converges uniformly? J. Foran [F] has given an example of a continuous function whose graph is not $\sigma$ porous. An affirmative answer would be given to our question if we could find such a function in $p B V$ for some $p \geq 1$ or even if we could find such a function in $H B V$.

\section{REFERENCES}

[CG] J. T. Cox and P. S. Griffin, How porous is the graph of Brownian motion?, Trans. Amer. Math. Soc. 325 (1991), 119-140.

[F] J. Foran, Continuous function need not have $\sigma$ porous graphs, Real Anal. Exchange 11 (1985-1986), 194-203.

[G] C. Goffman, Everywhere convergence of Fourier series, Indiana Univ. Math. J. 20 (19701971), 107-112.

[R] W. Rogosinski, Fourier series, Chelsea, New York, 1950.

[W] D. Waterman, On convergence of Fourier series of functions of generalized bounded variation, Studia Math. 44 (1972), 107-117.

[Y] L. C. Young, Sur une généralization de la notion de variation de puissance p-ième bornée au sense de N. Wiener, et sur la convergence de séries de Fourier, C. R. Acad. Sci. Paris 204 (1937), 470-472.

Department of Mathematics, Purdue University, West Lafayette, Indiana 47907-1395 